Pacific Journal of Mathematics

A HARNACK ESTIMATE FOR REAL NORMAL SURFAC 


\title{
A HARNACK ESTIMATE FOR REAL NORMAL SURFACE SINGULARITIES
}

\author{
William A. Adkins
}

\begin{abstract}
According to Harnack's theorem the number of topological components of the real part of a nonsingular projective curve $X$ defined over $\mathbf{R}$ is at most $g(X)+1$, where $g(X)$ is the genus of $X$. The purpose of the present paper is to present two estimates which can be considered analogs of Harnack's theorem for normal surface singularities defined over $\mathbf{R}$.
\end{abstract}

1. Introduction. A simple example will suffice to illustrate the type of result which one may expect. Suppose $A \subseteq \mathbf{P}^{2}(\mathbf{C})$ is a projective plane curve defined over $\mathbf{R}$ and let $A_{\mathbf{R}}$ be the real part of $A$. Let $V \subseteq \mathbf{C}^{3}$ be the cone over $A$ and let $\left(V_{\mathbf{R}}, 0\right)$ be the germ at 0 of the real part of $V$. Then $\left(V_{\mathbf{R}}, 0\right)$ is connected, but the punctured variety $\left(V_{\mathbf{R}} \backslash\{0\}, 0\right)$ may have two components for each connected component of $A_{\mathrm{R}}$. Thus the number of components of $\left(V_{\mathbf{R}} \backslash\{0\}, 0\right)$ is bounded by $2+2 g(A)=b_{0}(A)+b_{1}(A)$ $+b_{2}(A)$ where $b_{i}(A)$ is the $i$ th betti number of $A$. If one resolves the singularity $(V, 0)$, the exceptional curve $E$ is just the curve $A$, so we conclude that the number of components of $\left(V_{\mathbf{R}} \backslash\{0\}, 0\right)$ is bounded by the sum of the betti numbers of the exceptional curve in a resolution of $(V, 0)$. It is in precisely this form that one may obtain a Harnack estimate for an arbitrary normal surface singularity defined over $\mathbf{R}$. Specifically, let $(V, p)$ be a normal surface singularity defined over $\mathbf{R}$ and let $\pi: M \rightarrow V$ be the minimal normal resolution of $V$ with exceptional curve $E=\pi^{-1}(p)$. Then the following three results will be proved.

1.1. TheOREM. $\pi: M \rightarrow V$ is a real resolution, i.e. it is defined over $\mathbf{R}$.

1.2. TheOrem. $b_{0}\left(V_{\mathbf{R}} \backslash\{0\}, 0\right) \leq \sum_{i=0}^{2} b_{i}(E)$.

1.3. TheOREM. By Theorem $1.1, E$ is defined over $\mathbf{R}$ and there is the estimate $b_{0}\left(E_{R}\right) \leq 1+p_{g}(E)$ where $p_{g}(E)$ is the geometric genus of $E$.

After recalling some definitions and preliminary results in $\$ 2$, Theorem 1.1 is proved in $\S 3$, while $\S 4$ contains the proofs of the two Harnack estimates. 
2. Preliminaries. All complex spaces are assumed to be reduced, second countable and pure dimensional. By surface we will mean a complex space of dimension two. Let $V$ be a normal surface and let $\pi$ : $M \rightarrow V$ be a resolution of $V$, i.e. $M$ is nonsingular, $\pi$ is proper and $\pi$ : $M \backslash \pi^{-1}(S(V)) \rightarrow V \backslash S(V)$ is biholomorphic, where $S(V)$ denotes the singular set of $V$. The minimal resolution of $V$ is the unique resolution through which all other resolutions factor. This can be obtained from an arbitrary resolution by successively contracting exceptional curves of the first kind (Laufer [6] page 87). A normal resolution of $V$ is a resolution in which the exceptional curve has nonsingular components which intersect transversely and no three components intersect. There is a unique minimal normal resolution obtained from the minimal resolution by means of quadratic transforms [6] page 91 .

Let $A=\bigcup_{i=1}^{k} A_{i}$ be a curve with irreducible components $A_{i}$. Associated to $A$ is a graph $G$, called the dual graph of $A$, formed as follows. The vertices of $G$ are the irreducible components $A_{i}$ of $A$, and each point of $A_{i} \cap A_{j}$ gives an edge joining the vertices $A_{i}$ and $A_{j}$. If $V$ is a normal surface and $\pi: M \rightarrow V$ is a resolution, then the dual graph of the resolution is the dual graph of the exceptional curve.

The exceptional curves of normal resolutions will be used frequently, so we give them a name. An $N$-curve is a projective curve in which the irreducible components are nonsingular, intersect transversely, and no three components intersect. The topology of an $\mathrm{N}$-curve is completely determined by the topology of the irreducible components and the dual graph, as in the following result, which is easily proved by a MayerVietoris argument (or see Brenton [2]). For homology we will always use $\mathbf{Z}_{2}$ coefficients. Thus $b_{i}(X)=\operatorname{dim}_{\mathbf{Z}_{2}} H_{i}\left(X, \mathbf{Z}_{2}\right)$. Then

1.2. Proposition. Let $A=\bigcup_{i=1}^{k} A_{i}$ be an $N$-curve with dual graph $G$.

$$
\begin{aligned}
& b_{1}(A)=\sum_{i=1}^{k} b_{1}\left(A_{i}\right)+b_{1}(G), \\
& b_{2}(A)=k
\end{aligned}
$$

$$
p_{g}(A)=\operatorname{dim}_{\mathbf{C}} H^{1}\left(A, \mathcal{O}_{A}\right)=\sum_{i=1}^{k} g\left(A_{i}\right)+b_{1}(G),
$$

where $g\left(A_{i}\right)$ denotes the genus of the nonsingular curve $A_{l}$.

The basic estimate we shall use in our proofs is the following "Smith theory" inequality. 
2.2. Theorem. Let $X$ be a finite cell complex and $T: X \rightarrow X a$ continuous involution with fixed point set $F$. Then

$$
\operatorname{dim} H_{*}(F) \leq \operatorname{dim} H_{*}(X) .
$$

The symbol $\operatorname{dim} H_{*}$ ( ) refers to the sum of the betti numbers. For the proof of this result see Wilson [9] page 72.

3. Real resolutions. A complex space with conjugation is a complex space $X$ together with an antiholomorphic involution $\sigma: X \rightarrow X$. The fixed point set of $\sigma$ is called the real part of $X$ and will be denoted $X_{\mathbf{R}}$. If $(X, \sigma)$ and $(Y, \tau)$ are complex spaces with conjugations, then a holomorphic map $f: X \rightarrow Y$ is said to be real if $\tau \circ f=f \circ \sigma$. Thus $f\left(X_{\mathbf{R}}\right) \subseteq Y_{\mathbf{R}}$.

3.1. THEOREM. Let $(V, \sigma)$ be a normal surface with conjugation and let $\pi: M \rightarrow V$ be the minimal resolution of $V$. Then $M$ has a conjugation $\tau$ such that $\pi$ is a real map.

Proof. It will first be proved that there is some real resolution of $V$. According to a classical theorem of Zariski (see Lipman [7]) a resolution of each singular point of $V$ can be obtained by means of a finite sequence of quadratic transformations at singular points, followed by normalizations. Each of these two operations will be considered separately.

3.2. LEMMA. Let $(W, \sigma)$ be a reduced complex space with conjugation and let $\theta: W^{\prime} \rightarrow W$ be the normalization. Then there is a conjugation $\tau$ on $W^{\prime}$ with respect to which $\theta$ is a real map.

Proof. $\theta: W^{\prime} \backslash \theta^{-1}(S(W)) \rightarrow W \backslash S(W)$ is an analytic isomorphism so define $\tau$ on $W^{\prime} \backslash \theta^{-1}(S(W))$ by $\tau=\theta^{-1} \circ \sigma \circ \theta$. If $p \in S(W)$ then $\theta^{-1}(p)$ is in one-to-one correspondence with the irreducible components of the germ $(W, p)$. Since $\sigma$ must give a bijection between the irreducible components of $(W, p)$ and the irreducible components of $(W, \sigma(p))$, use this bijection to define $\tau: \theta^{-1}(p) \rightarrow \theta^{-1}(\sigma(p))$.

Now consider conjugations under quadratic transforms. Thus let $(W, \sigma)$ be a (normal) complex space with conjugation. Then $S(W)$ is invariant under $\sigma$. Let $p \in S(W)$. Then $\sigma(p) \in S(W)$ and there are two cases which will be considered separately.

$$
\text { 3.3. Case I. } p \in W_{\mathbf{R}} \text {, i.e. } \sigma(p)=p \text {. }
$$


In this case a holomorphic imbedding $(W, p) \subseteq\left(\mathbf{C}^{n}, 0\right)$ may be chosen which is conjugation invariant. Recall that if $\Gamma \subseteq\left(\mathbf{C}^{n}, 0\right) \times \mathbf{P}^{n-1}(\mathbf{C})$ is defined by

$$
\Gamma=\left\{\left(\left(z_{1}, \ldots, z_{n}\right),\left[w_{1}, \ldots, w_{n}\right]\right): z_{i} w_{j}=z_{j} w_{i} \quad \text { for } 1 \leq i, j \leq n\right\}
$$

then $\mathbf{C}^{n} \backslash\{0\} \subseteq \Gamma$ and the quadratic transform of $(W, p)$ is the closure of $W \backslash p$ in $\Gamma$. Since $\Gamma$ is defined by real equations and $W \backslash p$ is conjugation invariant, it follows that the strict transform of $(W, p)$ is also conjugation invariant and this gives an extension of $\sigma$ to the quadratic transform of $W$ at $p$.

\subsection{Case II. $\sigma(p) \neq p$.}

In this case one may choose an imbedding of $(W, p)$ in $\left(\mathbf{C}^{n}\right.$, $(i, 0, \ldots, 0))$ via holomorphic coordinates $\xi_{1}, \ldots, \xi_{n}$. Then $\overline{\xi_{i} \circ \sigma}(1 \leq i \leq n)$ are holomorphic coordinates on $(\sigma W, \sigma(p))$ which give an imbedding of $(\sigma W, \sigma(p))$ into $\left(\mathbf{C}^{n},(-i, 0, \ldots, 0)\right)$. Thus there is a commutative diagram

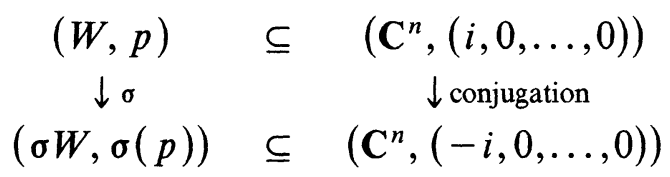

Now perform simultaneous quadratic transforms at $(i, 0, \ldots, 0)$ and $(-i, 0, \ldots, 0)$. It is then clear from the construction that the strict transform of $(W, p)$ is taken via conjugation to the strict transform of $(\sigma W, \sigma(p))$. Hence $\sigma$ extends to a conjugation on the space obtained by doing simultaneous quadratic transforms at $p$ and $\sigma(p)$.

We now return to the proof of Theorem 3.1. By Zariski's theorem some resolution of $V$ will be obtained if one alternately does quadratic transformations and normalizations. If, in addition, one is careful to simultaneously do quadratic transformations at both $p$ and $\sigma(p)$, then (3.2)-(3.4) show that a real resolution $\pi^{\prime}:\left(M^{\prime}, \tau^{\prime}\right) \rightarrow(V, \sigma)$ is obtained. By Theorem 5.9 (page 87) of Laufer [6], the minimal resolution of $V$ is obtained from $M^{\prime}$ by successively collapsing exceptional curves of the first kind in $M^{\prime}$. But the condition for a curve to be exceptional of the first kind is purely topological (genus 0 and self intersection -1). Thus if $A$ is exceptional of the first kind, then $\tau^{\prime}(A)$ is also exceptional of the first kind, and if one simultaneously collapses $A$ and $\tau^{\prime}(A)$, (this is possible because $A \cap \tau^{\prime}(A)=\varnothing$ by negative-definiteness of exceptional sets) a 
new surface is obtained which also has a conjugation map. Since one eventually arrives at the minimal resolution of $V$ by this process, the proof of 3.1 is complete.

3.5. RemarKs. (1) Further applications of (3.3) and (3.4) show that the minimal normal resolution of $V$ also supports a conjugation with respect to which the resolution map is real.

(2) If $(V, 0) \subseteq\left(\mathbf{C}^{n}, 0\right)$ is a conjugation invariant variety and $\pi: M \rightarrow V$ is a real resolution, then the exceptional curve $\pi^{-1}(0)$ may have no real points. A necessary and sufficient condition for $\pi^{-1}(0)_{\mathbf{R}}$ to be nonempty is that $\left(V_{\mathbf{R}}, 0\right) \nsubseteq(S(V), 0)$. See [5] for an algebraic version of this result. In the analytic case it is an easy consequence of the properness of $\pi$ : $M \rightarrow V$. For example, the cone $z_{1}^{2}+z_{2}^{2}+z_{3}^{2}=0$ is resolved by a single quadratic transformation at 0 and the exceptional curve is the rational curve $w_{1}^{2}+w_{2}^{2}+w_{3}^{2}=0$ in $\mathbf{P}^{2}(\mathbf{C})$ which is conjugation invariant, but which has no real points.

4. Harnack estimates. If $(X, p)$ is the germ of a topological space at $p$ then $b_{i}(X, p)$ denotes the $i$ th betti number of a sufficiently small representative of the germ $(X, p)$ near $p$.

4.1. THEOREM. Let $(V, p)$ be a normal surface singularity with conjugation and let $\pi: M \rightarrow V$ be a real resolution of $V$ with exceptional curve $E$. Then

$$
b_{0}\left(V_{\mathbf{R}} \backslash\{p\}, p\right) \leq \sum_{i=0}^{2} b_{i}(E)
$$

Proof. If $V_{\mathbf{R}}=\{p\}$ the inequality is trivially satisfied since the left hand side is 0 . Thus assume that $V_{\mathbf{R}} \neq\{p\}$. Let $X=M_{\mathbf{R}}$ and $A=E_{\mathbf{R}}$. Then $V_{\mathbf{R}} \backslash\{p\} \simeq X \backslash A$ so it suffices to compute $b_{0}(X \backslash A)$. First note that $H_{0}(X, X \backslash A)=0$. This is because $A$ is one dimensional and every connected component of $X$ has dimension 2. Thus every connected component of $X$ intersects $X \backslash A$. Also $H_{1}(X, X \backslash A) \simeq H^{1}(A)$ by Alexander duality (Spanier [8], page 296). (All homology and cohomology is computed with $\mathbf{Z}_{2}$ coefficients.)

The exact homology sequence of the pair $(X, X \backslash A)$ contains the segment $H_{1}(X, X \backslash A) \rightarrow H_{0}(X \backslash A) \rightarrow H_{0}(X) \rightarrow 0$. Thus $b_{0}(X \backslash A) \leq$ $b_{0}(X)+b_{1}(A)$. But it is easy to see that if one chooses a sufficiently small neighborhood of $p$, then the resulting $X$ will satisfy $b_{0}(X)=b_{0}(A)$. (Simply triangulate $M$ so that $X, E$, and $A$ are all subcomplexes and then 
take a sufficiently fine barycentric subdivision.) Since $A=E_{\mathrm{R}}$ an application of Theorem 2.2 gives

$$
b_{0}(X \backslash A) \leq b_{0}(A)+b_{1}(A) \leq \sum_{i=0}^{2} b_{i}(E) \text {. }
$$

4.2. REMARK. The example presented in the introduction shows that the estimate in Theorem 4.1 is probably the best that can be obtained. For a concrete example, the cone $V=\left\{z^{2}=x^{2}+y^{2}\right\} \subseteq \mathbf{C}^{3}$ will have $b_{0}\left(V_{\mathbf{R}} \backslash\{0\}\right)=2$ while $E$ is the projective line so the sum of the betti numbers of $E$ will also be 2 .

Let $(V, p)$ be a real normal surface singularity and let $\pi: M \rightarrow V$ be a real resolution. Theorem 4.1 gives an estimate of the number of topological components of $V_{\mathbf{R}} \backslash\{p\}$. A second natural question is to ask for the number of topological components of the real part $E_{\mathbf{R}}$ of the exceptional curve $E$ of $(V, p)$. The next result gives such an estimate. It is essentially an extension of Harnack's theorem to curves which are not necessarily irreducible. The specific curves to be considered are the $N$-curves introduced in section 2 .

4.3. Theorem. Let $A=\bigcup_{i=1}^{k} A_{i}$ be a connected $N$-curve with a conjugation $\sigma$. Then $b_{0}\left(A_{\mathbf{R}}\right) \leq 1+p_{g}(A)$.

4.4. Remark. If $G$ is the dual graph of $A$, recall from Proposition 2.1 that the geometric genus $p_{g}(A)=\sum_{i=1}^{k} g\left(A_{i}\right)+b_{1}(G)$.

Proof. (of 4.3) The conjugation $\sigma$ determines an involution of the dual graph $G$ of the curve $A$ by sending the vertex of $G$ corresponding to the irreducible component $A_{i}$ of $A$ to the vertex corresponding to the irreducible component $\sigma\left(A_{i}\right)$. Consider first the special case in which $\sigma$ induces the identity on $G$, i.e. $\sigma\left(A_{i}\right)=A_{i}$ for $1 \leq i \leq k$. By the Smith theory inequality and Proposition 2.1,

$$
\begin{aligned}
b_{0}\left(A_{\mathbf{R}}\right)+b_{1}\left(A_{\mathbf{R}}\right) & \leq 1+b_{1}(A)+b_{2}(A) \\
& =1+2 \sum_{i=1}^{k} g\left(A_{i}\right)+b_{1}(G)+k \\
& =2+2 \sum_{i=1}^{k} g\left(A_{l}\right)+b_{1}(G)+(k-1) .
\end{aligned}
$$

Claim. $b_{1}\left(A_{\mathbf{R}}\right)-b_{0}\left(A_{\mathbf{R}}\right) \geq(k-1)-b_{1}(G)$. 
Substituting this inequality into formula (4.1) gives Theorem 4.3 in the special case in which $\sigma$ induces the identity on $G$. The claim will be verified by induction on $k$, the number of irreducible components of the curve $A$. If $k=1$ then $A_{\mathbf{R}}$ consists of a disjoint collection of circles so $b_{1}\left(A_{\mathbf{R}}\right)=b_{0}\left(A_{\mathbf{R}}\right)$ and the claim is satisfied in this case. Now let $A^{\prime}$ be an $N$-curve with $k-1$ irreducible components and suppose $A=A^{\prime} \cup A_{k}$. Let $G^{\prime}$ be the dual graph of $A^{\prime}$ and consider separately two cases.

Case 1. $b_{1}(G)=b_{1}\left(G^{\prime}\right)$.

In this case $A_{k} \cap A^{\prime}$ must consist of a single point $p$ and $p \in A_{\mathbf{R}}$. Thus a circle of $\left(A_{k}\right)_{\mathbf{R}}$ and a circle of $A_{\mathbf{R}}^{\prime}$ are connected at the point $p$, so that $b_{0}\left(A_{\mathbf{R}}\right)=b_{0}\left(A_{\mathbf{R}}^{\prime}\right)+b_{0}\left(\left(A_{k}\right)_{\mathbf{R}}\right)-1$ and $b_{1}\left(A_{\mathbf{R}}\right)=b_{1}\left(A_{\mathbf{R}}^{\prime}\right)+$ $b_{1}\left(\left(A_{k}\right)_{\mathbf{R}}\right)$. Hence

$$
b_{1}\left(A_{\mathbf{R}}\right)-b_{0}\left(A_{\mathbf{R}}\right)=b_{1}\left(A_{\mathbf{R}}^{\prime}\right)-b_{0}\left(A_{\mathbf{R}}^{\prime}\right)+1 \geq(k-1)-b_{1}(G) .
$$

Case 2. $b_{1}(G)>b_{1}\left(G^{\prime}\right)$.

It will always be true that $b_{0}\left(A_{\mathbf{R}}\right) \leq b_{0}\left(\left(A_{k}\right)_{\mathbf{R}}\right)+b_{0}\left(A_{\mathbf{R}}^{\prime}\right)$ and $b_{1}\left(A_{\mathbf{R}}\right)$ $\geq b_{1}\left(\left(A_{k}\right)_{\mathbf{R}}\right)+b_{1}\left(A_{\mathbf{R}}^{\prime}\right)$. Thus

$$
\begin{aligned}
b_{1}\left(A_{\mathbf{R}}\right)-b_{0}\left(A_{\mathbf{R}}\right) & \geq b_{1}\left(A_{\mathbf{R}}^{\prime}\right)-b_{0}\left(A_{\mathbf{R}}^{\prime}\right) \geq(k-2)-b_{1}\left(G^{\prime}\right) \\
& \geq(k-1)-b_{1}(G)
\end{aligned}
$$

since $b_{1}(G)>b_{1}\left(G^{\prime}\right)$.

Thus the claim is verified and hence Theorem 4.3 is proved in the case in which every irreducible component of $A$ is conjugation invariant.

Now consider a second special case. In this case $A$ will consist of two irreducible components $A_{1}$ and $A_{2}$ which are interchanged by the conjugation map $\sigma$. Then the fixed point set $A_{\mathbf{R}}$ consists of finitely many points which are contained in $A_{1} \cap A_{2}$. The dual graph $G$ of $A$ consists of 2 vertices joined by $e=\#\left(A_{1} \cap A_{2}\right)$ edges. Thus $b_{0}\left(A_{\mathbf{R}}\right) \leq e=1+b_{1}(G)$.

We now proceed with the general case. Thus let $A$ be a connected $N$-curve with conjugation $\sigma$ and with dual graph $G$. The involution $\sigma$ on $A$ induces an involution $T$ on $G$. Extending $T$ to be a simplicial map on the topological space $G$, Theorem 2.2 may be applied to conclude that

$$
b_{0}(F)+b_{1}(F) \leq 1+b_{1}(G)
$$

where $F$ is the fixed point set of $T$. The fixed points of $T$ are of two distinct types. Type I are the vertices of $G$ fixed by $T$ (i.e. the irreducible components of $A$ which are invariant under the conjugation $\sigma$ ) together with the edges joining fixed vertices. The fixed points of type II are the centers of the edges joining two adjacent vertices which are interchanged by $T$. 
Let $C_{1}, \ldots, C_{r}$ be the connected components of $F$ of type I and let $D_{1}, \ldots, D_{s}$ be the pairs of adjacent vertices of $G$ which are interchanged by $T$. Then corresponding to each $C_{J}$ is a connected curve $A_{C_{J}} \subseteq A$ whose irreducible components are the vertices of $C_{\text {J }}$. Each irreducible component of $A_{C}$ is conjugation invariant. Similarly, for each $D_{i}$ there is a connected curve $A_{D_{1}} \subseteq A$ consisting of two irreducible components which are interchanged by $\sigma$. Then

$$
A_{\mathbf{R}}=\bigcup_{j=1}^{r}\left(A_{C_{j}}\right)_{\mathbf{R}} \cup \bigcup_{l=1}^{s}\left(A_{D_{l}}\right)_{\mathbf{R}} .
$$

By the two special cases done above,

$$
\begin{aligned}
b_{0}\left(A_{\mathbf{R}}\right) & \leq r+\sum_{j=1}^{r} p_{g}\left(A_{C_{j}}\right)+s+\sum_{l=1}^{s} b_{1}\left(D_{l}\right) \\
& \leq \sum_{j=1}^{k} g\left(A_{J}\right)+b_{0}(F)+b_{1}(F) \\
& \leq \sum_{j=1}^{k} g\left(A_{\jmath}\right)+1+b_{1}(G)=1+p_{g}(A) .
\end{aligned}
$$

The third inequality comes from formula (4.2) while the second inequality comes from Proposition 2.1 and the fact that each $D_{l}$ contributes exactly $1+b_{1}\left(D_{l}\right)$ isolated fixed points to $F$ since that is exactly the number of edges joining the two vertices of $D_{i}$. Thus the proof of Theorem 4.3 is complete.

4.5. Remark. A special case is worth mentioning. Suppose that $A$ is a connected $N$-curve with a conjugation and assume that $H_{1}(A, \mathbf{R})=0$. Then $p_{g}(A)=0$ by Proposition 2.1 so in this case the theorem says that $A_{\mathbf{R}}$ is connected. This occurs for example if $A$ is the exceptional set in the resolution of a rational singularity (Artin [1], Brieskorn [4]). Furthermore, from the explicit formulas in Brieskorn [3], which are formulas with real coefficients, one sees that all of the rational double points admit conjugations.

\section{REFERENCES}

[1] M. Artin, On isolated rational singularities of surfaces, Amer. J. Math., 88 (1966), 129-136.

[2] L. Brenton, Some algebraicity criteria for singular surfaces, Inventiones Math., 41 (1977), 129-147.

[3] E. Brieskorn, Über die auflösung gewisser singularitäten von holomorphen abbildungen, Math. Annalen, 166 (1966), 76-102. 
[4] _ Rationale singularitäten komplexer flächen, Inventiones Math., 4 (1968), 336-358.

[5] G. Efroymson, Local reality on algebraic varieties, J. Algebra, 29 (1974), 133-142.

[6] H. Laufer, Normal Two-dimensional Singularities, Princeton U. Press, Princeton, N.J. (1971).

[7] J. Lipman, Rational singularities, with applications to algebraic surfaces and unique factorization, Institut des Hautes Etudes Scien. 36 (1969), 195-280.

[8] E. Spanier, Algebraic Topology, McGraw-Hill, New York (1966).

[9] G. Wilson, Hilbert's sixteenth problem, Topology, 17 (1978), 53-73.

Received October 16, 1982 and in revised form January 5, 1984.

Louisiana State University

BATON ROUGE, LA 70803 



\section{PACIFIC JOURNAL OF MATHEMATICS EDITORS}

\author{
DONALD BABBITT (Managing Editor) \\ University of California \\ Los Angeles, CA 90024 \\ J. DugundJI \\ University of Southern California \\ Los Angeles, CA 90089-1113 \\ R. FINN \\ Stanford University \\ Stanford, CA 94305 \\ HERMANN FLASChKa \\ University of Arizona \\ Tucson, AZ 85721
}

C. C. MOORE

University of California

Berkeley, CA 94720

Arthur Ogus

University of California

Berkeley, CA 94720

Hugo RossI

University of Utah

Salt Lake City, UT 84112

H. SAMELSON

Stanford University

Stanford, CA 94305

ASSOCIATE EDITORS
R. ARENS
E. F. BECKENBACH
B. H. NeUmanN
F. WOLF
K. YosHIDA (1906-1982)

\section{SUPPORTING INSTITUTIONS}
UNIVERSITY OF ARIZONA
UNIVERSITY OF BRITISH COLUMBIA
CALIFORNIA INSTITUTE OF TECHNOLOGY
UNIVERSITY OF CALIFORNIA
MONTANA STATE UNIVERSITY
UNIVERSITY OF NEVADA, RENO
NEW MEXICO STATE UNIVERSITY
OREGON STATE UNIVERSITY

\author{
UNIVERSITY OF OREGON \\ UNIVERSITY OF SOUTHERN CALIFORNIA \\ STANFORD UNIVERSITY \\ UNIVERSITY OF HAWAII \\ UNIVERSITY OF TOKYO \\ UNIVERSITY OF UTAH \\ WASHINGTON STATE UNIVERSITY \\ UNIVERSITY OF WASHINGTON
}

The Supporting Institutions listed above contribute to the cost of publication of this Journal, but they are not owners or publishers and have no responsibility for its content or policies.

Mathematical papers intended for publication in the Pacific Journal of Mathematics should be in typed form or offset-reproduced (not dittoed), double spaced with large margins. Please do not use built up fractions in the text of the manuscript. However, you may use them in the displayed equations. Underline Greek letters in red, German in green, and script in blue. The first paragraph must be capable of being used separately as a synopsis of the entire paper. In particular it should contain no bibliographic references. Please propose a heading for the odd numbered pages of less than 35 characters. Manuscripts, in triplicate, may be sent to any one of the editors. Please classify according to the scheme of Math. Reviews, Index to Vol. 39. Supply name and address of author to whom proofs should be sent. All other communications should be addressed to the managing editor, or Elaine Barth, University of California, Los Angeles, California 90024.

There are page-charges associated with articles appearing in the Pacific Journal of Mathematics. These charges are expected to be paid by the author's University, Government Agency or Company. If the author or authors do not have access to such Institutional support these charges are waived. Single authors will receive 50 free reprints; joint authors will receive a total of 100 free reprints. Additional copies may be obtained at cost in multiples of 50 .

The Pacific Journal of Mathematics is issued monthly as of January 1966. Regular subscription rate: $\$ 190.00$ a year (5 Vols., 10 issues). Special rate: $\$ 66.00$ a year to individual members of supporting institutions.

Subscriptions, orders for numbers issued in the last three calendar years, and changes of address should be sent to Pacific Journal of Mathematics, P.O. Box 969, Carmel Valley, CA 93924, U.S.A. Old back numbers obtainable from Kraus Periodicals Co., Route 100, Millwood, NY 10546.

The Pacific Journal of Mathematics at P.O. Box 969, Carmel Valley, CA 93924 (ISSN 0030-8730) publishes 5 volumes per year. Application to mail at Second-class postage rates is pending at Carmel Valley, California, and additional mailing offices. Postmaster: Send address changes to Pacific Journal of Mathematics, P.O. Box 969, Carmel Valley, CA 93924.

PUBLISHED BY PACIFIC JOURNAL OF MATHEMATICS, A NON-PROFIT CORPORATION

Copyright $\odot 1984$ by Pacific Journal of Mathematics 


\section{Pacific Journal of Mathematics}

Vol. 114, No. $2 \quad$ June, 1984

William Allen Adkins, A Harnack estimate for real normal surface

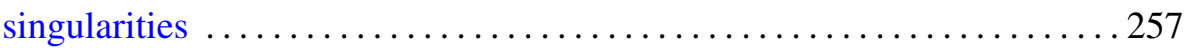

George E. Andrews, Multiple series Rogers-Ramanujan type identities . . . . 267

Didier Arnal, $*$ products and representations of nilpotent groups . . . . . . 285

David Cox and Walter Raymond Parry, Representations associated with

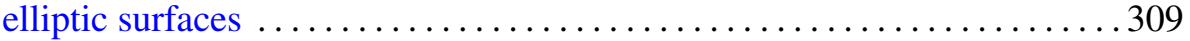

Joanne Marie Dombrowski, Tridiagonal matrix representations of cyclic

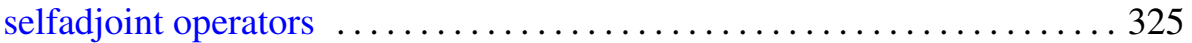

Ronald Dotzel, An Artin relation $(\bmod 2)$ for finite group actions on

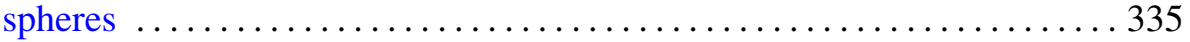

Leo Egghe, Convergence of adapted sequences of Pettis-integrable

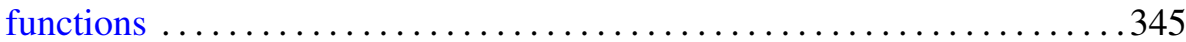

Rebecca A. Herb, Characters of induced representations and weighted

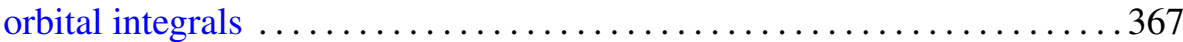

Steven M. Kahn, Cobordism obstructions to fibering manifolds over

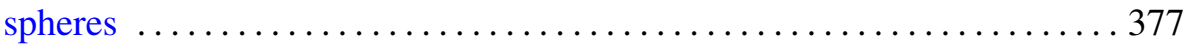

Robert D. Little, Projective space as a branched covering of the sphere with orientable branch set

Claude Schochet, Topological methods for $C^{*}$-algebras. III. Axiomatic

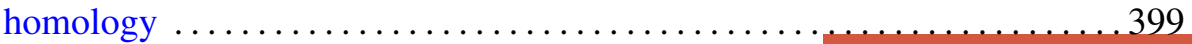

Claude Schochet, Topological methods for $C^{*}$-algebras. IV. $\bmod p$ homology

James M. Stormes, On the $K O$-orientability of complex projective varieties

Josephine Anne Ward, Characterization of homogeneous spaces and their norms 